\title{
ON THE EFFECT OF DISTRIBUTED CAPACITY IN SINGLE LAYER SOLENOIDS.
}

\author{
By J. C. Hubbard.
}

$\mathrm{T}^{\mathrm{H}}$ HE present paper describes a precise method of measuring the periods of electric oscillations, from the results of which is determined the effective, or distributed capacity of coils of a single layer over a wide range of coil dimensions. The method is checked by a determination, using the measured periods and the calculated inductances and external capacities, of the quantity $v$, the ratio of the electromagnetic to the electrostatic unit of quantity.

The problem of a coil oscillating in its own free period has proved one of the most difficult in mathematical physics. In addition to its inductance the coil possesses an effective capacity resulting from the fact that different parts of the coil are at different periodically varying potentials. There has been no theory developed except for coils of a single layer, and the theory which has been so developed agrees only roughly and within restricted limits with the experimental data. Drude ${ }^{1}$ developed expressions for the wave-length and capacity of a freely oscillating coil and measured the wave-lengths of coils over a wide range of coil dimensions. A more rigorous treatment has been given by W. Len $z^{2}$ who developed expressions for the wave-lengths of extremely long and of extremely short coils and covered the region between by interpolation.

Another method of finding the free periods consists in measuring the period of a circuit composed of an inductance and an external capacity in series. The square of the period should be a linear function of the external capacity. Thus, $T^{2}=4 \pi^{2} L\left(K+K_{0}\right)$, where $K$ is the capacity of the external variable condenser, and $K_{0}$ is the remaining capacity of the system, composed in part of the distributed capacity of the coil. $K_{0}$ may thus be obtained by extrapolation. Such measurements were made by Professor Webster ${ }^{3}$ who verified the linear relation. Experi-

1 P. Drude, Ann. d. Phys., (4), 9, pp. 293-339, I902.

2 W. Lenz, Ann. d. Phys., (4), 43, pp. 749-797, I9r4. See also, K. W. Wagner, Das Eindringen einer elektromagnetischen Welle in eine Spule mit Windungskapazität, Elektrotechnik u. Maschinenbau, Wien, Nos. 8 and 9, I9I5; Die Theorie des Kettenleiters nebst Anwendungen, Archiv für Elektrotechnik, Nos. Io and II, I9I 5 .

${ }^{3}$ A. G. Webster, An Experimental Determination of the Period of Electrical Oscillations, Phys. Rev., VI., pp. 297-3I4, I898. 
ments along this line were projected by the writer in I9II, making use of a new method for measuring periods, with the special object of determining with accuracy the effective capacity of the coils used. Results were presented before the American Physical Society in October, I9I2, and in April, I915." 1

In the meantime Professor G. W. O. Howe ${ }^{2}$ in a paper on the calibration of wave-meters had shown the linear relation to hold down to the free period of the coil and that the effect of the self-capacity of the coil is to add a constant capacity to that of the system.

W. Rietz, ${ }^{3}$ making use of a Helmholtz pendulum traced the wave form of an oscillating circuit with variable capacity and came to the same conclusion. His results, for the one single-layer coil studied, give only the order of magnitude of the distributed capacity. Frederick A. Kolster ${ }^{4}$ has shown how the distributed capacity of a coil may be found from the apparent inductances at various frequencies.

The present paper is an attempt to supplement the work of Drude, each coil being part of an oscillating circuit of the sort common in wireless telegraphy and instruments, one end of the coil being earthed, and not, as in Drude's experiments, oscillating freely as in the Tesla transformer.

\section{Measurement of Period.}

The method here used for measuring periods has already been briefly described by the writer. ${ }^{5}$ It makes use of a modification of the apparatus used earlier by Professor Webster and owes its precision largely to the excellent design of his drop chronograph. The modification introduced by the writer consists of an arrangement by means of which an electrometer is made to give deflections of a sign and magnitude depending upon the potential at any desired instant after the interruption of the current in an inductive circuit. If the circuit be oscillating the device enables the oscillations of potential to be traced and furnishes a very precise means of measuring the period of oscillations.

Lever $I$ (Fig. I) closes the circuit through the inductance $L$, the amount of current being regulated by the non-inductive resistance $R$. The condenser $K$ is connected across the inductance terminals. A weight is released by an electromagnet and falls freely through a definite vertical distance striking lever $I$ and opening the circuit. Oscillations ensue, the period of which depends upon the system $K, L$ and connections, the

${ }^{1}$ J. C. Hubbard, Phys. Rev., N.S., I., pp. 247-249, I9r3, VI., p. 58, I9r5.

2 G. W. O. Howe, Proc. Phys. Soc. Lond., XXIV., pp. 25I-259, I9I2.

${ }^{3}$ W. Rietz, Ann. d. Phys., (4), 4r, pp. 543-569, I9I3.

${ }^{4}$ F. A. Kolster, Proc. Inst. Radio Eng., I., No. 2, pp. 19-34, I913.

${ }^{5}$ Loc. cit. 
variable resistance and battery being cut out and earth-connected at $E$. At a definite moment later the projectile strikes lever 2 which, in turn strikes lever 3 , lifting it from the contact $P$. Lever 2 is permanently connected with one plate of the air condenser and may be regarded as a potential terminal. The contact $P$ is connected with one pair of electrometer quadrants which are disconnected from earth at the moment of release of projectile. As may be seen, connection is established and immediately broken between the electrometer at the moment that lever 2 strikes 3 . The phase of oscillation at this moment determines the sign and magnitude of the charge received by the electrometer. The duration of the time of contact between condenser and electrometer must be consid-

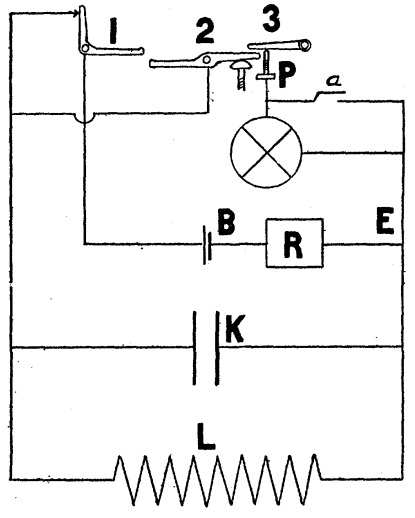

Fig. 1. ered as finite, but the results show that it is either extraordinarily constant or very small compared to the periods used.

The system comprising levers 2 and 3 and the contact screw $P$, in addition to a catch (not shown) to prevent lever 3 from returning to $P$ during the electrometer deflection, is mounted on a heavy plate of ebonite which is screwed to a carriage moved vertically by a micrometer screw reading to $0.001 \mathrm{~mm}$. In practice it is customary to determine frequently the highest position for the carriage at which deflections begin to be observed in the electrometer. This is the point at which contact is made with the electrometer at the same instant as the breaking of the circuit and is taken as the micrometer zero. At this point the projectile has a velocity depending upon its height of fall above lever $I$. Subsequent readings of the micrometer, referred to this point as origin, thus measure the further excursion of the projectile, from which its average velocity during any interval that oscillations are taking place, and hence the time required, may be found. In making the determination of a period it is customary to locate positions for two successive nodes, then to look for a node at twice this distance, and so on, doubling each time, until a suitable number of nodes, sometimes several hundred, have been covered. The initial and final nodal positions of the screw are determined to $0.0001 \mathrm{~cm}$., and a range of not less than $0.5 \mathrm{~cm}$. is covered, giving an accuracy in the length measurement better than one part in two thousand. The period of oscillation is then $T=s \ln V$, where $s$ is the distance on the micrometer screw corresponding to $2 n$ 
half waves, and $V$ is the average velocity of projectile during the oscillations which are being measured. It appeared that there were four possible sources of error in the determination of $V$, each tending to make the actual value less than that found; viz., residual magnetism in the electromagnetic release of the projectile, air resistance, moment of inertia of the levers, and tension of the rubber bands holding the levers in place. By using various values of magnetization in the electromagnet, various velocities up to $325 \mathrm{~cm}$./ $/ \mathrm{sec}$., with consequent variation of air resistance, by trying levers of different dimensions and under different degrees of tension, it is found that these errors are not of a magnitude, singly or collectively, to be detected in the results.

The falling weight, or projectile, is that of Professor Webster's drop chronograph, a description of which has been published. ${ }^{1}$

\section{The Electrical System.}

The solenoid, the distributed capacity of which is to be measured, is mounted, axis horizontal, at a distance of several feet from the condenser. The leads from the solenoid to the condenser form a rectangular circuit of known dimensions (Fig. 2), the plane of which passes through the axis

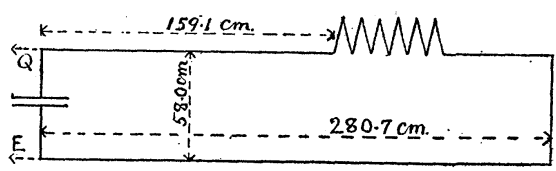

Fig. 2.

of the coil. The upper side of the rectangle is a continuation of the generating line of the coil surface on its lower side, the wire of the coil at the ends making a right angle turn about a peg set into the core. The wire $Q$, leads to contact with levers. $I$ and 2 .

The air condenser consists of two plates of ground and polished steel held apart by sets of three small glass cylinders of length determined by an optical micrometer. Six sets of cylinders furnish the capacities (Kirchhoff's formula) given in Table III.

Tables I. and II. give the dimensions and calculated values of inductance of the accurately constructed coils. Rosa has given tables from which Nagaoka's formula for the inductance of a current sheet may be corrected to represent the actual inductance of a coil. The following data are necessary: $:^{2} r=$ mean radius of coil, $b=$ length from center to center of $n+\mathrm{I}$ turns $=\left(h-d^{\prime}\right) n /(n-\mathrm{I})$, where $h=$ axial length of

${ }^{1}$ Loc. cit.

${ }^{2}$ E. B. Rosa, Bull. Bu. Standards, 8, No. I, p. Ir9, I9r2-13. 
the coil from outside to outside of insulated wire of diameter $d^{\prime} . \quad d=$ diameter of bare wire, $D=$ pitch of winding $=\left(h-d^{\prime}\right) /(n-\mathrm{r})$. The inductance of the rectangular circuit is calculated from Rosa's formula, ${ }^{1}$ in which $a=$ length, $b=$ breadth of rectangle, and $\rho=$ radius of bare

TABLE I

Data for Coils.

\begin{tabular}{c|r|c|c|c|c|c|c|c|c}
\hline Series. & $r \mathrm{~cm}$. & $d \mathrm{~cm}$. & $d^{\prime} \mathbf{c m}$. & $D / d$ & Series. & $r \mathbf{c m}$. & $d \mathbf{c m}$. & $d^{\prime} \mathbf{c m}$. & $D / d$ \\
\cline { 2 - 8 } $\mathrm{E}$ & 9.740 & .0650 & .0840 & 1.50 & $\mathrm{~K}$ & 6.297 & .0650 & .0840 & 1.40 \\
$\mathrm{~F}$ & 21.163 & .1291 & .1529 & 1.63 & $\mathrm{~L}$ & 31.57 & .0636 & .0844 & 4.98 \\
$\mathrm{G}$ & 9.84 & .0625 & .0834 & 3.13 & $\mathrm{M}$ & 9.64 & .0627 & .0840 & 5.06 \\
$\mathrm{H}$ & 31.63 & .1810 & .2095 & 1.75 & $\mathrm{~N}$ & 9.745 & .0627 & .0840 & 1.56 \\
$\mathrm{I}$ & 31.55 & .0632 & .0830 & 5.02 & $\mathrm{O}$ & 21.165 & .0650 & .0860 & 3.26 \\
$\mathrm{~J}$ & 7.96 & .0659 & .0855 & 1.35 & & & & & \\
\hline
\end{tabular}

TABLE II.

Dimensions and Inductances of Coils.

\begin{tabular}{|c|c|c|c|c|c|c|c|}
\hline Series. & $n$. & $h$. & $L^{\prime} \times 10^{-6} \mathrm{Cm}$ & Series. & $n$. & $h$. & $L^{\prime} \times 10^{-6} \mathrm{Cm}$. \\
\hline $\mathrm{E}_{1}$ & 886 & 86.549 & 30.904 & $\mathrm{H}_{3}$ & 70 & 22.06 & 3.839 \\
\hline $\mathrm{E}_{2}$ & 750 & 73.263 & 25.732 & $\mathrm{H}_{4}$ & 50 & 15.75 & 2.273 \\
\hline$E_{3}$ & 625 & 61.050 & 20.993 & $\mathrm{H}_{5}$ & 35 & 10.95 & 1.283 \\
\hline $\mathrm{E}_{4}$ & 500 & 48.837 & 16.279 & $\mathrm{I}_{1}$ & 162 & 51.19 & 12.926 \\
\hline $\mathrm{E}_{5}$ & 375 & 36.624 & 11.609 & $\mathrm{I}_{2}$ & 100 & 31.50 & 6.551 \\
\hline $\mathrm{E}_{6}$ & 250 & 24.412 & $7.0 \dot{4} 03$ & $\mathrm{I}_{3}$ & 70 & 21.97 & 3.850 \\
\hline$F_{1}$ & 215 & 45.37 & 12.637 & $\mathrm{I}_{4}$ & 50 & 15.58 & 2.288 \\
\hline $\mathrm{F}_{2}$ & 180 & 37.97 & 9.993 & $\mathrm{I}_{5}$ & 35 & 10.84 & 1.292 \\
\hline $\mathrm{F}_{3}$ & 145 & 30.58 & 7.442 & $\mathrm{~J}$ & 216 & 19.2 & 4.41 \\
\hline $\mathrm{F}_{4}$ & 110 & 23.18 & 5.035 & $\mathrm{~K}$ & 619 & 56.20 & 9.694 \\
\hline$F_{5}$ & 85 & 17.90 & 3.448 & $\mathrm{~L}$ & 162 & 51.17 & 12.946 \\
\hline $\mathrm{F}_{6}$ & 60 & 12.61 & 2.027 & $M$ & 264 & 83.61 & 2.796 \\
\hline $\mathrm{G}_{1}$ & 436 & 85.10 & 7.763 & $\mathrm{~N}$ & 868 & 84.88 & 30.202 \\
\hline $\mathrm{G}_{2}$ & 300 & 58.51 & 5.126 & $\mathrm{O}_{1}$ & 215 & 45.33 & 12.674 \\
\hline $\mathrm{H}_{1}$ & 161 & 50.92 & 12.861 & $\mathrm{O}_{2}$ & 180 & 37.94 & 10.021 \\
\hline $\mathrm{H}_{2}$ & 100 & 31.61 & 6.539 & $\mathrm{O}_{3}$ & 145 & 30.54 & 7.465 \\
\hline
\end{tabular}

wire. The wire of the coils of series $E$ to $O$ was double cotton covered. Coils $E$ to $I$ and $L$ to $O$, inclusive, were wound on built up cores of white pine with the grain parallel to the axis of the coil. Coil $K$ was wound on a tube of ebonite of $12.5 \mathrm{~cm}$. external, and I0.I $\mathrm{cm}$. internal diameter, and of length, $60.5 \mathrm{~cm}$. All cores except that of series $J$ were given a light thread in the lathe barely sufficient to hold the tightly wound wire in place. Coil $J$ was closely wound on a glass cylinder of thin walls (2 mm.).

The total inductance, $L$, of the system is obtained by adding the induc${ }^{1}$ Loc. cit., p. 155 . 
tance of the coil, $L^{\prime}$, given in Table II., to that of the rectangular circuit, $L^{\prime \prime}$. This would, of course, be strictly true only if the terminals of the coils had been led to the axis before coming off, but the change is so slight as not to appear in any figures retained for total inductance.

Preliminary experiments were conducted to test the effect of changing the dimensions of the rectangular lead circuit and the relative position of coil and condenser. There was no sensible difference in the value of $K_{D}$ or of $v$ obtained with the same coil in the three circuits used. The dimensions of the circuit used in the final experiments are given in Fig. $2, \rho$ being $0.025 \mathrm{I} \mathrm{cm}$., and the inductance of the circuit, $L^{\prime \prime}=10,260 \mathrm{~cm}$.

\section{RESULTS.}

The period of oscillation of the electrical system is, by reason of the small resistance in the oscillating circuit and the relatively small capacity, represented by $T=(2 \pi / v) \sqrt{L C}$, where $L$ is the combined inductance of coil and rectangular circuit, and $C$ is the combined capacity of the system, consisting of the air condenser $K$, the capacity of leads and connections $K_{L}$, and the distributed capacity of the coil, $K_{D}$. Thus,

$$
T^{2}=\frac{4 \pi^{2} L}{v^{2}}\left(K+K_{L}+K_{D}\right) .
$$

If there is no appreciable variation of $L$ or of $K_{D}$ with the frequency, $T^{2}$ will be a linear function of $K$, or $T^{2}=\alpha K+\beta$, where $\alpha=4 \pi^{2} L / v^{2}$, and $\beta=\left(4 \pi^{2} L / v^{2}\right)\left(K_{L}+K_{D}\right)$. The constants $\alpha$ and $\beta$ may be determined by the method of least squares, giving

$$
\beta / \alpha=K_{L}+K_{D}=K_{0}, \quad \text { and } \quad v=2 \pi \sqrt{L / \alpha} .
$$

Table III. is chosen to illustrate the manner in which these quantities are determined, and is an example of the best work done with the drop chronograph, though a large majority of the thirty-four similar tables

TABLE III.

Determination of $\alpha$ and $\beta$ : Series $E_{2}$.

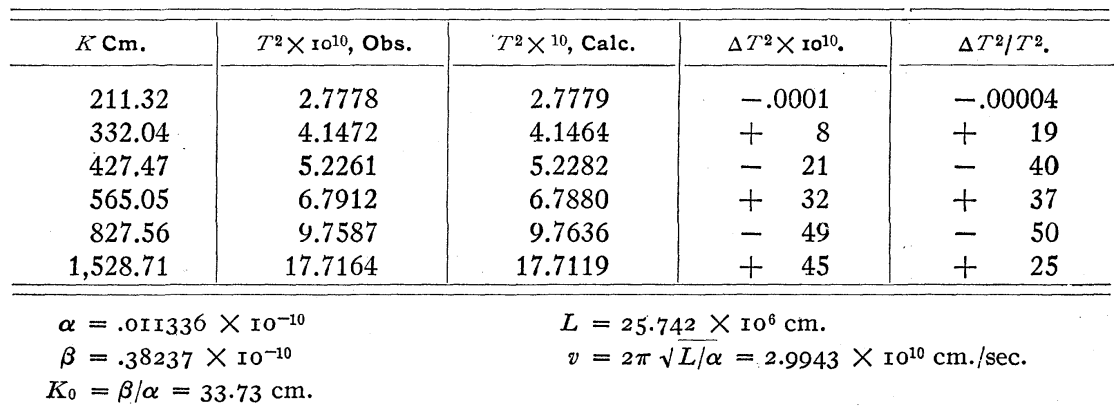


at hand show an accuracy as great, and in only two cases, owing to trouble with the drop chronograph, did the average value of $\Delta T^{2} / T^{2}$ exceed one tenth per cent. Table III. illustrates very effectively that, to a high. degree of precision, there is neither a variation of inductance or of distributed capacity in the coils as here used.

Having determined $K_{0}$ as in Table III., it is next necessary to determine $K_{L}$, the capacity of leads, in order that the distributed capacity, $K_{D}$, of the coil may be found. The capacity of leads has been directly measured by an electrostatic method. ${ }^{1}$ This is not a satisfactory procedure, as it is necessary in making such a measurement to disconnect the condenser and coil from the lead wires, tending to raise somewhat the capacity of the leads. A more satisfactory method consists in finding values of $K_{0}$ for geometrically similar coils of different linear dimensionsSuppose one coil is of linear dimensions $n$ greater than another. Accord. ing to the well-known theorem of electrically similar systems the distributed capacity of the larger coil should be $n$ times that of the smaller. Thus $n\left(K_{D}\right)_{1}=\left(K_{D}\right)_{n}$, whence $(\beta / \alpha)_{1}=K_{L}+\left(K_{D}\right)_{1}$, and $(\beta / \alpha)_{n}=K_{L}$ $+n\left(K_{D}\right)_{1}$, from which an independent determination of $K_{L}$ and $K_{D}$ is possible. If we consider coils of the same core material and of the same ratio of pitch to diameter of bare wire, we may put, after Drude, $K_{D}=r \phi(h / 2 r)$, whence $K_{0}=\beta / \alpha=K_{L}+r \phi(h / 2 r)$. From graphs of $K_{0}$ as a function of $h / 2 r$ (see Tables I., II. and IV.) for the series E, F and $\mathrm{H}$, which are strictly comparable, we may determine $K_{0}$ for the corresponding values of $h / 2 r$. For $h / 2 r=\mathrm{I}$, for example, we have,

$$
\begin{array}{ll}
\mathrm{E}, & K_{0}=32.15=K_{L}+9.74 \phi, \\
\mathrm{F}, & K_{0}=44.85=K_{L}+2 \mathrm{I} .16 \phi, \\
\mathrm{H}, & K_{0}=54.5^{2}=K_{L}+31.63 \phi,
\end{array}
$$

from which $K_{L}=22.25 \mathrm{~cm}$. (See inserted figure in upper right hand corner of Fig. 4.) The value measured directly by the electrostatic method is $23.4 \mathrm{~cm}$., an excellent agreement, being slightly larger, as is to be expected. We then have, $K_{D}=K_{0}-22.25$. The results are given in Table IV.

It is evident from the results that the factor of chief importance in determining the distributed capacity is the radius of the coil, in all cases the two quantities being of the same order. This agrees with Drude's theory, insofar as it concerns the radius alone, but the form of his expression for distributed capacity does not hold in the present case, the coils here being earthed at one end.

${ }^{1}$ J. C. Hubbard and H. F. Stimson, Phys. Rev., N.S., I., pp. 245-247, I9r3. 

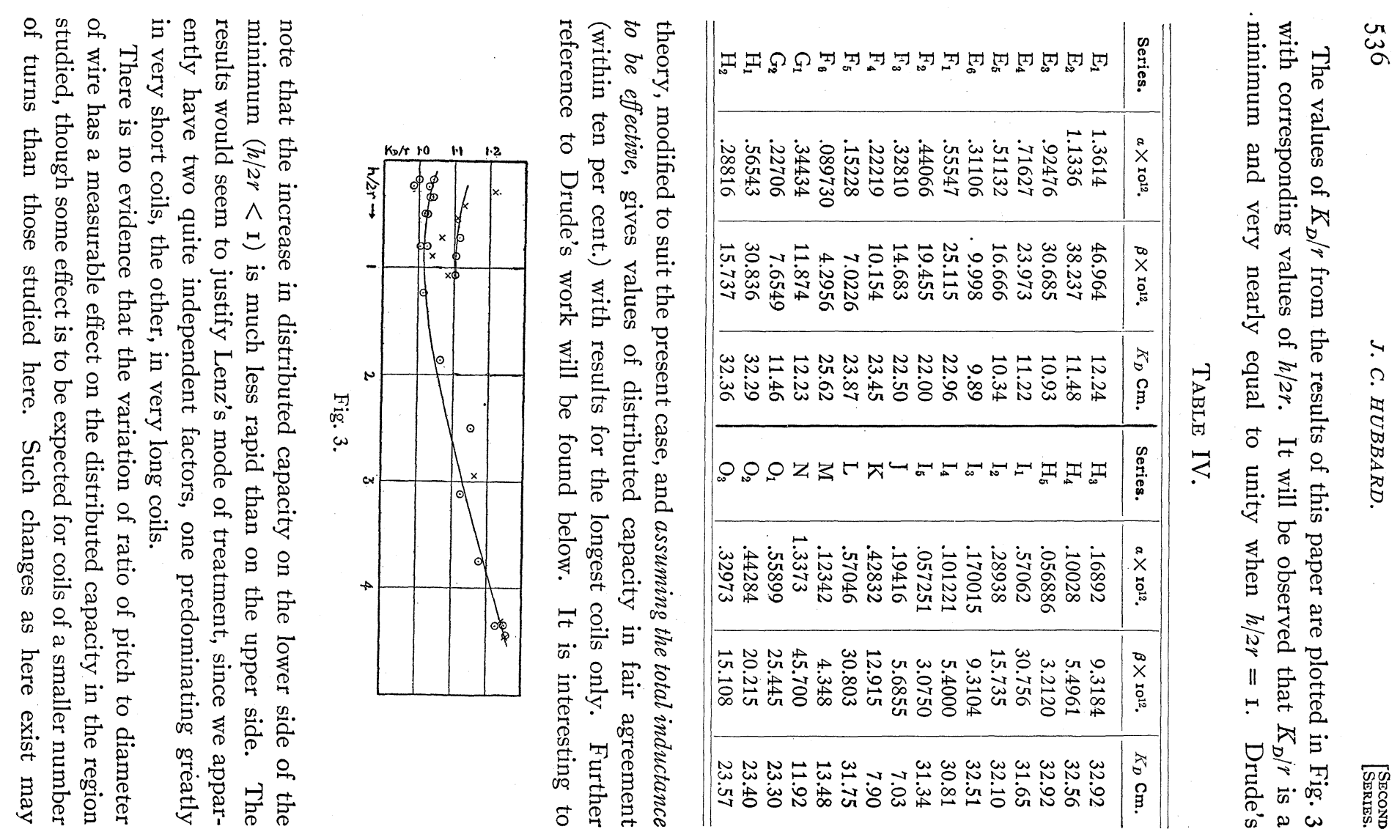
quite certainly be attributed to slight changes in the position of the wire leading from the condenser to the lever system.

The nature of the core in the present experiments seems to be of relatively small importance in the magnitude of distributed capacity. For example, the ebonite tube, Series $\mathrm{K}$, gives, for $h / 2 r=4.46$, a value of $K_{D} / r=\mathrm{I} .254$, while for coil $E_{1}$, solid wooden core, $h / 2 r=4.44$ and $K_{D} / r=1.257$, a difference well within such accidental errors as might occur. On the other hand, Series J, close wound glass cylinder, $h / 2 r$ $=\mathrm{I} .205$, gives $K_{D} / r=.884$, a result twelve per cent. smaller than the corresponding value for wooden cores, but, on filling the cylinder with kerosene the value is raised by only one per cent.; in the cases of both the empty and filled cylinder the values lie much closer to those for corresponding coils on wooden cores than do Drude's results for freely oscillating coils on glass cylinders.

In the present experiments, accordingly, the dielectric nature of the core is less important, owing to the fact, as before noted, that one end of the coil is connected to earth, producing a different configuration of the field of electric force from that in Drude's experiments. Fewer lines, relatively, would traverse the core.

To bridge the gap between these experiments and those of Drude the periods of two of the larger coils, with a threefold variation of radius, were measured with $K=0, i$. e., with the air condenser disconnected. Since $T^{2}=\alpha K+\beta$, we have $T_{0}=\sqrt{ } \bar{\beta}$. The results follow.

Series $\mathrm{L}, \alpha=.0057046 \times 10^{-10}, \sqrt{\beta}=.5554 \times \mathrm{ro}^{-5}, T_{0}$ observed $=.57223 \times 10^{-5}$.

Series $\mathrm{N}, \alpha=.013373 \times 1 \mathrm{IO}^{-10}, \sqrt{\beta}=.67602 \times 10^{-5}, T_{0}$ observed $=.70765 \times 10^{-5}$.

Let $\Delta$ be the small positive change introduced in the lead wire capacity by disconnecting the condenser. Then, $T^{2}=\alpha \Delta+\beta$, and $\Delta=\left(T_{0}{ }^{2}-\beta\right)$ $/ \alpha$, giving $\Delta=3.4 \mathrm{I} \mathrm{cm}$. for Series $\mathrm{L}$, and $3.28 \mathrm{~cm}$. for Series N. Taking as the mean, $\Delta=3.34 \mathrm{~cm}$. and calculating $T_{0}{ }^{2}=3.34 \alpha+\beta$, we have for calculated periods of $\mathrm{L}$ and $\mathrm{N}$ respectively, $.57 \mathrm{I} 8$ and $.7082 \times 10^{-5}$ sec., the corresponding observed periods being .5722 and $.7076 \times 10^{-5}$ sec.

This is a remarkably good agreement, and shows that the discrepancy between $T_{0}$ and $\sqrt{\beta}$ may entirely be explained by the change introduced in lead capacity. It may be concluded that, to a high degree of precision, the square of the period of a coil and condenser circuit is a linear function of the external capacity down to the value of the capacity of the coil.

This conclusion draws attention to a very interesting matter, also discussed in Howe's paper. In all our work we have assumed that the 
total inductance of the coil is effective. Drude assumes a distribution of current in a freely oscillating coil such that the total inductance is multiplied by the factor $2 / \pi$ to give the effective inductance. The effective capacity of the coil would then be $\pi / 2$ times the values we have given. Since the relation $T^{2}=\alpha K+\beta$, holds down to $K=0$, with precision, it would seem that either the total inductance must be effective in a freely oscillating coil, or there must be some exactly compensating change in the effective capacity.

The free periods of all the coils used, earthed at one end, have been calculated by putting $K=-K_{L}$, giving $T_{F}=-\alpha K_{L}+\beta$, and $\frac{1}{2} \lambda_{F}=\frac{1}{2} 3 \times{ }^{10}{ }^{10} T_{F}$. The results follow very closely Drude's expression $\frac{1}{2} \lambda=l f(h / 2 r, D / d, e)$ if we take for $l$ twice the length of wire in the coil, and for $f$ values in his tables corresponding to values of $h$ equal to twice the axial length of coil for coils without cores. The values of $f$ are plotted with corresponding values of $2 h / 2 r=h^{\prime} / 2 r$ in Fig. 4. (Series E and H,

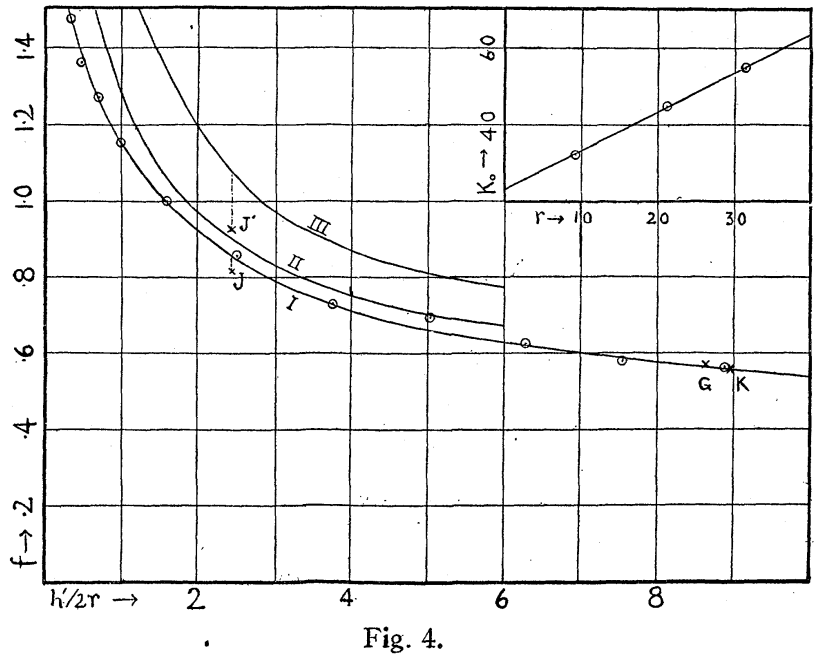

only, are plotted for the sake of clearness.) The smooth curve, I., drawn through these points, closely parallels Drude's curve, II., for similar coils without cores, in which $h^{\prime}=h$. The curve, III., is drawn from Drude's results for corresponding coils on wooden cores, and, as may be seen, lies much farther away from the results of the present paper. The point $K$, giving the value of $f$ for Series $\mathrm{K}$ (ebonite tube), is seen to correspond well with the results of this paper for wooden cores. From Drude's work a value considerably less would be expected. The departure of the value of $f$ for series $\mathrm{J}$ (coil wound on glass cylinder), from the curve for wooden cores is seen to be much smaller than the 
departure of Drude's corresponding value for a coil on a glass cylinder, $J^{\prime}$, from his results for similar coils on wooden cores. All these facts are in further support of the conclusion that, for coils earthed at one end the material of the core has much less effect on the magnitude of the distributed capacity than it has in coils freely oscillating with a potential node at the center.

\section{MeAsurement of $v$.}

As a check on the accuracy of the drop chronograph, a calculation of $v$, the ratio of the electromagnetic to the electrostatic unit of quantity, has been made from the results. We have,

from which,

$$
T^{2}=(2 \pi / v)^{2} L\left(K+K_{0}\right)=\alpha K+\beta,
$$

$$
v=2 \pi \sqrt{L / \alpha}
$$

Table III. gives an example of $v$ so computed. From the data already given $v$ may be calculated in each case. It will be found that the values are in excellent agreement. Owing to the greater care taken in the construction and measurement of dimensions of the coils of Series E and $\mathrm{F}$ we shall use the values of $v$ obtained in those series as examples. We have for the average deviations of $r$ and $h$, respectively; for $\mathrm{E}, .003$ and $.003 \mathrm{~cm}$.; for $\mathrm{F}, .008$ and $.005 \mathrm{~cm}$. Table V. gives the values of $v$, the computed deviation in $v, \Delta v_{c}$, as well as the actual deviation, $\Delta v_{0}$, from the mean.

TABLE V.

\begin{tabular}{|c|c|c|c|c|c|c|c|}
\hline Series. & $v \times 10^{-10}$ & $\Delta v_{c} \times 10^{-10}$ & $\Delta v_{0} \times \mathrm{ro}^{-10}$ & Series. & $v \times \mathrm{ro}^{-10}$ & $\Delta v_{c} \times 10^{-10}$ & $\Delta v_{0} \times 10^{-10}$. \\
\hline$E_{1}$ & 2.9942 & \pm .0013 & -.0008 & $F_{1}$ & 2.9983 & \pm .0013 & +.0030 \\
\hline$E_{2}$ & 43 & 10 & -7 & $F_{2}$ & 39 & 16 & - \\
\hline $\mathrm{E}_{3}$ & 50 & 11 & \pm & $\mathrm{F}_{3}$ & 52 & 13 & \pm \\
\hline $\mathrm{E}_{4}$ & 63 & 13 & 13 & $F_{4}$ & 43 & 16 & - \\
\hline $\mathrm{E}_{5}$ & 52 & 11 & +2 & $F_{5}$ & 45 & 14 & - \\
\hline $\mathrm{E}_{6}$ & 14 & - & - & $\mathrm{F}_{6}$ & 39 & - & - \\
\hline
\end{tabular}

Determination of $v$.

The values of $v$ for $E_{6}$ and $F_{6}$ are rejected because the smallness of periods in these cases made it impossible to carry out measurements with the smaller values of external capacity, giving values of $\alpha$ of a lower order of precision than in the other cases. From the first five results in each series, we have,

$$
\begin{array}{ll}
\mathrm{E}, & v=2.9950 \times 1 \mathrm{IO}^{10} \mathrm{~cm} . / \mathrm{sec} ., \\
\mathrm{F}, & v=2.9952 \times 10^{10} \mathrm{~cm} . / \mathrm{sec} .,
\end{array}
$$

with an accuracy better than one part in two thousand, a much higher degree of accuracy than has before been obtained by this method. This 
is the more interesting because the most authoritative writers on the subject have expressed the opinion that an accuracy greater than one part in one thousand could not be reached by the present method. ${ }^{1}$

The best determination of $v$ which has been made by any method is that by Rosa and Dorsey, ${ }^{2}$ who obtained the value, $v=2.9963 \times 10^{10}$ $\mathrm{cm} . / \mathrm{sec}$., with an accuracy of one part in ten thousand.

The values of $v$ here obtained are such as to commend the drop chronograph as an excellent instrument for the precise measurement of small intervals of time, for the calibration of wave-meters, and to establish the value of the present method of measuring periods. It would be possible, with small additional labor, to increase the accuracy of the time measurement to one part in ten thousand. This would give a value of $v$, using properly constructed coils, with a precision probably as great as that of Rosa and Dorsey.

The present method of measuring periods with the drop chronograph is especially useful for getting precise measures of small capacities or of variations of capacity, any capacity which has been introduced appearing as a change in $K_{0}$. In this way the capacities of lead wires, of electrometers, and the mutual effects of conductors have been studied. The apparatus as used in getting the results in this paper is sensitive to a change in $K_{0}$ of one part in one thousand, or, of .or to $.04 \mathrm{~cm}$. of capacity. It is obvious that the method is also useful for the precise determination of inductance, and should be especially valuable, because the capacity of the coil is eliminated.

Dielectric constants of insulating liquids have been measured by the writer. For the purpose a small air condenser in a suitable cell was introduced in series with $K$ and the period measured with it empty, or filled with the desired liquid. When conducting liquids are used the damping is so great that precise values of $T$ cannot be obtained by this method. A resonance method has accordingly been developed for the study of dielectric constants of conducting liquids. This method, using a circuit calibrated for wave-length by the drop chronograph, and an audion detector, is capable of yielding good results also for distributed capacity of coils.

Some work has been done on the distributed capacity of coils of more than one layer. This will appear in a later paper.

In conclusion it may be remarked that information is greatly needed concerning the distribution of current and potential in oscillating coils. Until such information is at hand it is probable that the theoretical treatment of the single layer solenoid will not make much headway.

${ }^{1}$ E. B. Rosa and N. E. Dorsey, Bull. Bu. Standards, 3, p. 6I 7, r907.

2 E. B. Rosa and N. E. Dorsey, Bull. Bu. Standards, 3, pp. 433-604, I907. 


\section{Summary.}

The present paper describes a precise method of measuring the electrical periods of circuits, from the results of which are calculated the effective, or distributed capacities of coils earthed at one end. The work covers a wide range of coil dimensions.

I. It is found that the relation between the square of the period and the external capacity is very accurately linear down to the free period of the coil. This relation is new only in the accuracy with which it has been proved.

2. That the distributed capacity of coils earthed at one end is not much affected by the nature of the core.

3. The distributed capacity of such coils is a minimum and practically numerically equal to the radius of coils of length equal to the diameter, increasing, for example, by twenty-five per cent. when the length of coil is four times its diameter. For the practical purposes of wireless telegraphy it would seem sufficiently accurate to consider the effective capacity of the coil as equal to its radius in $\mathrm{cm}$. of capacity, or to $(r / 9) \times 10^{-5}$ microfarads.

4. The results, extrapolated to the natural periods of the coils, for coils earthed at one end, and with wooden cores, are in good general agreement with the results of Drude for freely oscillating coils without cores and of twice the axial length. This gives additional support to conclusion (2).

5. The method is checked by a determination from the results, of $v$, the ratio of the electromagnetic to the electrostatic unit of quantity. Values are found having an accuracy better than one part in two thousand, establishing the reliability of the present method of measuring electrical periods and commending the drop chronograph as an instrument for the precise measurement of small intervals of time.

6. Some conclusions are drawn concerning the serviceability of the method for the absolute measurement of capacities, inductances, or dielectric constants.

7. It is difficult to reconcile the facts of this paper summarized in ( $\mathrm{I}$ ) and (5) with the general practise of assuming the effective inductance of an oscillating coil to be $2 / \pi$ times the total inductance. This question waits on more precise information as to the distribution of current and potential in a freely oscillating coil.

Collegiate Department,

Clark University,

August, I9r6 